\title{
OCCURRENCE OF HORDELYMUS EUROPAEUS (L.) JESS. EX HARZ (POACEAE) IN THE WIELKOPOLSKA REGION
}

\author{
Renata Nowińska, Aneta Czarna
}

\begin{abstract}
R. Nowińska, A. Czarna, Department of Botany, Poznań University of Life Sciences, Wojska Polskiego 71 C, 60-625 Poznań, Poland, e-mail: nowinska@up.poznan.pl, czarna@up.poznan.pl
\end{abstract}

(Received: September 7, 2015. Accepted: October 28, 2015)

\begin{abstract}
AвSTRACT. The paper provides information about new locality and the current distribution of Hordelymus europaeus in the Wielkopolska region. On the basis of a field study we present a comprehensive description of species locations, characterise inhabited communities, as well as the population size and morphological differences of wood barley in particular populations.
\end{abstract}

KEY WORDs: Hordelymus europaeus, Wielkopolska, rare species, Poaceae

\section{INTRODUCTION}

Wood barley (Hordelymus europaeus (L.) Jess. ex Harz) is perennial grass from the Triticeae tribe (MIZIANTY \& SZCZEPANIAK 1997). Culms of this species are erect, $40-130 \mathrm{~cm}$ long. Nodes and sheaths are hairy. Leaves have falcate auricles and a short membranous ligule. Inflorescence - the single and erect raceme - is $4-12 \mathrm{~cm}$ long. A cluster of tree spikelets is located at each node. Spikelets are up to ca $30 \mathrm{~mm}$ long from the base to the top of the very long lemma awn and comprise 1-2 fertile florets (KLImKo et al. 2015).

Wood barley shows a dispersed pattern of geographical distribution. It is found in extensive parts of Europe, with the clear orientation to Central and South Europe. It also grows in Asia Minor, as well as Northeast Africa (Meusel et al. 1965). In Central Europe wood barley is a character species of the beechwood with the alliance Fagion sylvaticae R. Tx. et Diem. 1936 (OBERDORfer 1990). Its distribution visibly corresponds with the range of beech, however it is also encountered in oak-hornbeam forests and in other forest communities (Mizianty 2001).

Species is considered as a rare or very rare in Poland (Mizianty 2001). Most localities are situated in lower areas of the Carpathian Mountains and the Sudeten Mountains (ZaJĄC \& ZaJĄC 2001, BARĆ \& Brzustewicz 2006). The moderately numerous localities are reported in north-eastern Poland and very few localities are reported in the Pomerania and
Kuyavia regions (ZAJĄC \& ZAjĄC 2001, MiZIAnTY et al. 2006). Till now, the species has been reported from only three localities in Wielkopolska, that is from a forest near Kąty, the Bytyń Forest as well as from Zielonagóra forest district (MizIANTY 2001). It is listed as a critically endangered species in this region (JACKOWIAK et al. 2007).

In this note we provide a new locality of wood barley in Wielkopolska, as well as verify the former localities with detailed description of the position and coordinates. We characterise communities with $H$. europaeus and provide general information about population of this species.

\section{MATERIAL AND METHODS}

Field observations were carried out in 2014 and 2015. The historical localities of $H$. europaeus were verified and a new locality was found. Phytosociological relevés were prepared using the Braun-Blanquet method. The nomenclature of vascular plants followed MireK et al. (2002), whereas that of mosses followed Ochyra et al. (2003). The names of the syntaxa and list of character species were given after Matuszkiewicz (2001). In each locality all shoots of wood barley were counted and 30 mature and most diverse shoots were measured. Measurements focused on the following features: height of a shoot, length of inflorescence, length and width of the middle leaf. 
The herbarium material is deposited at the Department of Botany, the Poznań University of Life Sciences (POZNB).

\section{RESULTS}

\section{NEW LOCALITY}

1. Łopuchówko Forest District, Buczyna Forest Subdistrict

Hordelymus europaeus was found in division 113 of the Buczyna Forest Subdistrict (coordinate $16^{\circ} 58^{\prime} \mathrm{E}$, $52^{\circ} 40^{\prime} \mathrm{N}$ ). The division is situated ca $0.5 \mathrm{~km} \mathrm{SE}$ of Słomowo and ca $4 \mathrm{~km}$ NE of the previously described locality in the Długa Goślina Forest Subdistrict. Wood barley specimens occur in the brown-mull Beech wood Galio odorati-Fagetum and form two agglomerations ca 150-200 m apart and ca $50 \mathrm{~m}$ from the forest border (Fig. 1). Agglomeration 1B occupies the seed tree stand. Due to harvesting of singular trees herb layer is relatively low-shaded and turf patches with a high participation of Poa nemoralis occur (Table 1).

Agglomeration 1A consists of ca 700 shoots, whereas $1 \mathrm{~B}$ consists of ca 450 shoots. Singular specimens occupy the area between both agglomerations. Shoots with inflorescence range from $37 \mathrm{~cm}$ to 128 $\mathrm{cm}$, racemes range from $4.5 \mathrm{~cm}$ to $11 \mathrm{~cm}$, leaf length was between $10 \mathrm{~cm}$ and $32 \mathrm{~cm}$ while leaf width 0.4 $\mathrm{cm}$ and $1 \mathrm{~cm}$ (Table 2 ).

\section{PREVIOUSLY DESCRIBED LOCALITIES}

2. Łopuchówko Forest District, Długa Goślina Forest Subdistrict

Locality was published by Mizianty (2001) and based on unpublished data of KorCZEWSKA that had been collected in 1985. In literature it is recorded with a code "Kąty".

Current search shows, that the species occurs in division 153 of the Długa Goślina Forest Subdistrict
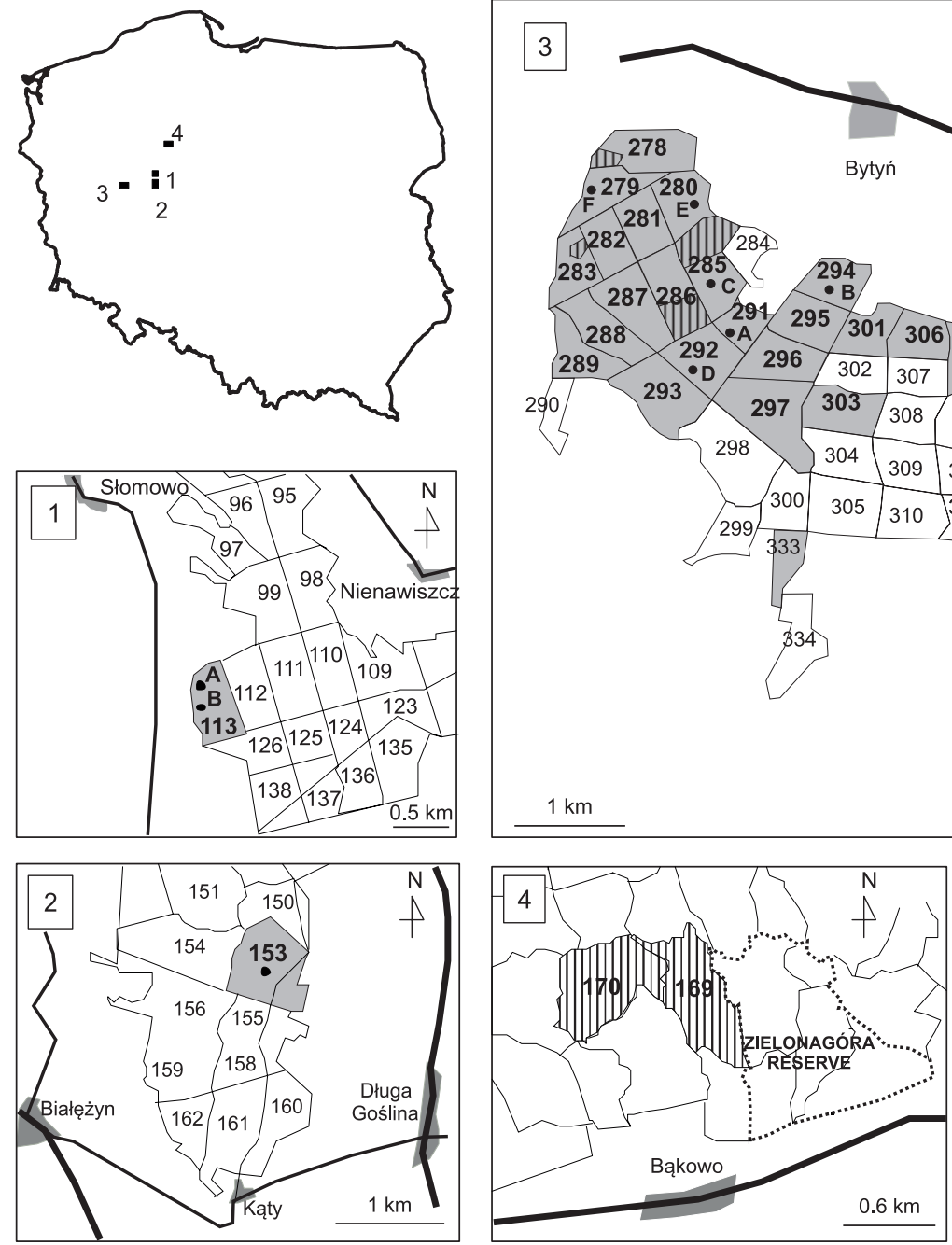

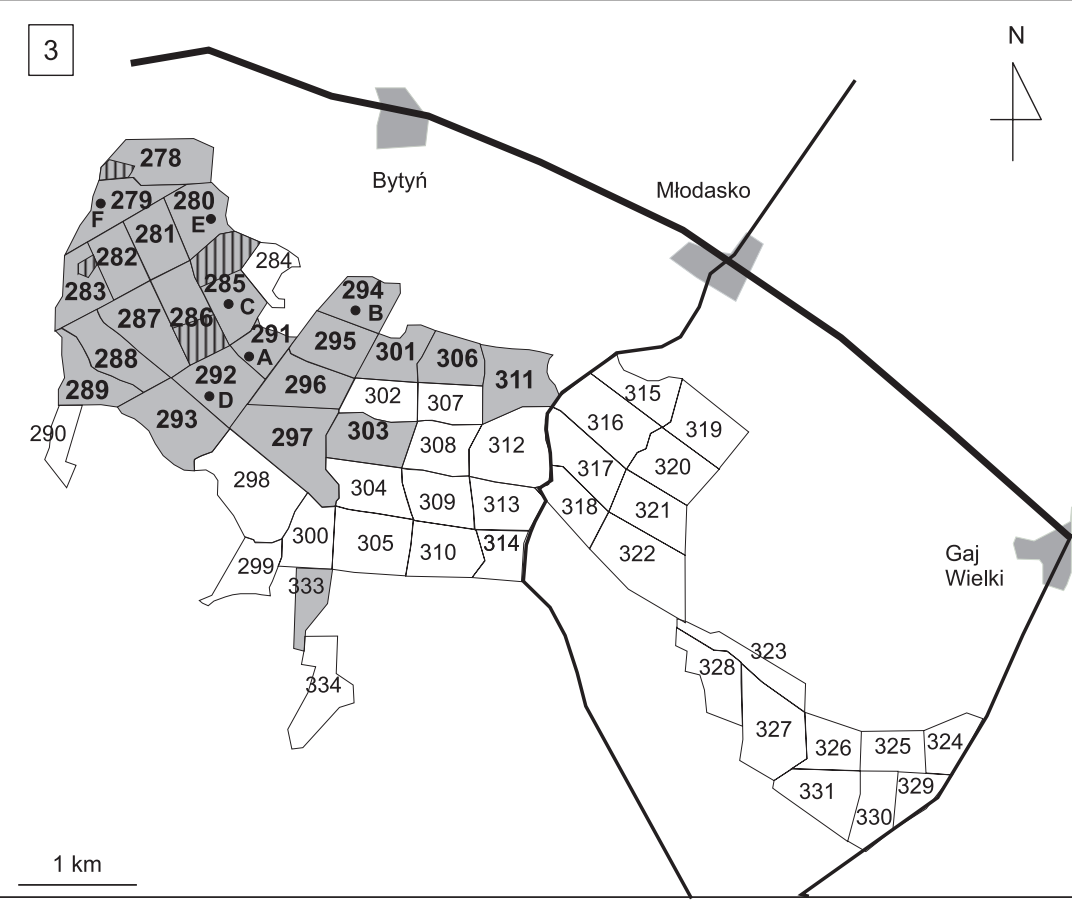

- Place where relevé was taken

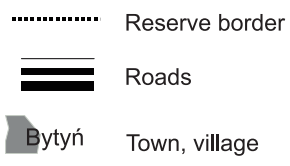

Fig. 1. Distribution of Hordelymus europaeus in the Wielkopolska region. Maps include the current division numbers 
Table 1. Plant communities with Hordelymus europaeus in the Buczyna (Bu), Długa Goślina (DG) and Bytyń (By) Forest Subdistricts. To check up the position of particular relevés (1A, 1B etc.) see Figure 1

\begin{tabular}{|c|c|c|c|c|c|c|c|c|c|}
\hline Successive number of relevé & 1 & 2 & 3 & 4 & 5 & 6 & 7 & 8 & 9 \\
\hline Position of locality & $1 \mathrm{~A}$ & $1 \mathrm{~B}$ & 2 & $3 \mathrm{~A}$ & $3 \mathrm{~B}$ & $3 \mathrm{C}$ & $3 \mathrm{D}$ & $3 \mathrm{E}$ & $3 \mathrm{~F}$ \\
\hline Data & $\begin{array}{c}10.08 \\
2014\end{array}$ & $\begin{array}{c}10.08 \\
2014\end{array}$ & $\begin{array}{c}05.08 . \\
2015 \\
\end{array}$ & $\begin{array}{c}25.08 . \\
2014\end{array}$ & $\begin{array}{c}25.08 . \\
2014\end{array}$ & $\begin{array}{c}25.08 . \\
2014\end{array}$ & $\begin{array}{c}25.08 . \\
2014 \\
\end{array}$ & $\begin{array}{c}27.08 . \\
2015 \\
\end{array}$ & $\begin{array}{r}27.08 \\
2015 \\
\end{array}$ \\
\hline Forest Subdistrict & $\mathrm{Bu}$ & $\mathrm{Bu}$ & DG & By & By & By & By & By & By \\
\hline Divisions & 113 & 113 & 153 & 291 & 294 & 285 & 292 & 280 & 279 \\
\hline Area of relevé $\left(\mathrm{m}^{2}\right)$ & 800 & 400 & 400 & 500 & 1000 & 1000 & 100 & 500 & 200 \\
\hline Cover of tree layer al (\%) & 80 & 80 & 20 & 80 & 80 & 70 & 70 & 10 & 50 \\
\hline Cover of tree layer a2 (\%) & 0 & 0 & 0 & 30 & 60 & 10 & 0 & 65 & 0 \\
\hline Cover of shrub layer b (\%) & 0 & 0 & ex & 5 & ex & ex & ex & 15 & 0 \\
\hline Cover of herbaceous layer c (\%) & 75 & 50 & 65 & 65 & 50 & 30 & 40 & 50 & 80 \\
\hline Cover of moss layer d (\%) & ex & ex & 5 & ex & ex & ex & ex & ex & ex \\
\hline Number of species & 33 & 36 & 40 & 27 & 36 & 39 & 41 & 33 & 37 \\
\hline I. Hordelymus europaeus (Fagion) & 2.3 & 2.3 & + & + & + & + & 1.1 & 1.1 & 2.2 \\
\hline
\end{tabular}

II. Fagion sylvaticae (incl. Galio odorati-Fagetum*)

\begin{tabular}{|c|c|c|c|c|c|c|c|c|c|}
\hline Fagus sylvatica (a1) & 4.3 & 3.3 & . & . & . & . & . & . & . \\
\hline Fagus sylvatica (c) & 1.1 & $\mathrm{r}$ & 1 & . & . & . & . & . & . \\
\hline Melica uniflora* & . & 2.3 & . & 1.1 & 3.3 & 1.1 & 1.1 & 2.3 & + \\
\hline \multicolumn{10}{|c|}{ III. Carpinion betuli (incl. Galio-Carpinetum*) } \\
\hline Carpinus betulus (a1) & . & 2.1 & . & 4.4 & 2.2 & . & 3.3 & . & . \\
\hline Carpinus betulus (a2) & . & . & . & 3.3 & 4.3 & 2.2 & . & 2.2 & . \\
\hline Carpinus betulus (c) & . & + & . & 1.1 & . & . & . & $\mathrm{r}$ & 2.3 \\
\hline Dactylis polygama & + & + & 2.2 & 1.1 & + & + & + & 1.1 & + \\
\hline Stellaria holostea & . & . & . & . & . & 1.1 & 1.1 & + & + \\
\hline Galium sylvaticum* & . & . & . & $\mathrm{r}$ & . & $\mathrm{r}$ & . & . & . \\
\hline Chaerophyllum temulum* & . & $\mathrm{r}$ & $\mathrm{r}$ & . & . & + & + & . & . \\
\hline \multicolumn{10}{|l|}{ IV. Querco-Fagetea } \\
\hline Acer pseudoplatanus (a1) & . & . & 2 & 2.2 & . & . & . & . & . \\
\hline Acer pseudoplatanus (c) & . & . & + & 2.2 & . & . & . & $\mathrm{r}$ & . \\
\hline Acer platanoides (c) & . & . & . & . & + & . & . & $\mathrm{r}$ & . \\
\hline Corylus avellana (c) & . & . & . & . & + & + & . & . & $\mathrm{r}$ \\
\hline Euonymus europaea (c) & . & . & . & . & + & . & . & + & . \\
\hline Sorbus torminalis (c) & . & . & . & . & $\mathrm{r}$ & . & . & . & $\mathrm{r}$ \\
\hline Brachypodium sylvaticum & . & . & $\mathrm{r}$ & $\mathrm{r}$ & $\mathrm{r}$ & + & + & + & . \\
\hline Festuca gigantea & . & . & . & . & $\mathrm{r}$ & $\mathrm{r}$ & $\mathrm{r}$ & . & $\mathrm{r}$ \\
\hline Carex sylvatica & . & . & . & + & . & $\mathrm{r}$ & . & & . \\
\hline Poa nemoralis & 3.3 & 1.2 & $\mathrm{r}$ & . & . & . & . & . & . \\
\hline Dryopteris filix-mas & . & $\mathrm{r}$ & 1 & . & $\mathrm{r}$ & . & . & 1.1 & . \\
\hline Galeobdolon luteum & + & 2.3 & . & . & . & . & . & . & . \\
\hline Galium odoratum & + & + & . & 2.2 & 1.1 & . & . & + & $\mathrm{r}$ \\
\hline Milium effusum & + & 1.2 & . & . & + & + & . & $\mathrm{r}$ & + \\
\hline Pulmonaria obscura & . & . & . & + & + & + & . & . & . \\
\hline Stachys sylvatica & $\mathrm{r}$ & . & . & + & + & . & 1.1 & + & + \\
\hline Viola reichenbachiana & + & + & + & + & . & + & $\mathrm{r}$ & . & $\mathrm{r}$ \\
\hline Circaea lutetiana & . & $\mathrm{r}$ & . & $\mathrm{r}$ & $\mathrm{r}$ & $\mathrm{r}$ & $\mathrm{r}$ & $\mathrm{r}$ & $\mathrm{r}$ \\
\hline Atrichum undulatum & $\mathrm{r}$ & $\mathrm{r}$ & & & & & & $\mathrm{r}$ & $\mathrm{r}$ \\
\hline \multicolumn{10}{|l|}{ V. Artemisietea vulgaris } \\
\hline Cirsium arvense & . & . & $\mathrm{r}$ & . & . & . & + & . & $\mathrm{r}$ \\
\hline Urtica dioica & 1.3 & 1.2 & 2 & + & + & + & 1.1 & $\mathrm{r}$ & + \\
\hline Fallopia dumetorum & + & + & $\mathrm{r}$ & . & . & . & . & $\mathrm{r}$ & . \\
\hline Rubus caesius & . & . & . & . & . & + & 1.1 & $\mathrm{r}$ & 2.2 \\
\hline Galeopsis pubescens & $\mathrm{r}$ & $\mathrm{r}$ & 1 & . & $\mathrm{r}$ & $\mathrm{r}$ & + & $\mathrm{r}$ & . \\
\hline Alliaria petiolata & $\mathrm{r}$ & . & 1 & . & . & + & . & . & . \\
\hline Chelidonium majus & $\mathrm{r}$ & $\mathrm{r}$ & $\mathrm{r}$ & . & . & . & . & . & . \\
\hline Geum urbanum & . & . & $\mathrm{r}$ & . & $\mathrm{r}$ & $\mathrm{r}$ & . & . & $\mathrm{r}$ \\
\hline Geranium robertianum & + & $\mathrm{r}$ & + & . & $\mathrm{r}$ & . & + & $\mathrm{r}$ & $\mathrm{r}$ \\
\hline
\end{tabular}




\begin{tabular}{|c|c|c|c|c|c|c|c|c|c|}
\hline Impatiens parviflora & + & 1.1 & 2 & + & . & + & 1.1 & . & + \\
\hline Lapsana communis & $\mathrm{r}$ & . & $\mathrm{r}$ & $\mathrm{r}$ & $\mathrm{r}$ & . & + & $\mathrm{r}$ & . \\
\hline \multicolumn{10}{|c|}{ VI. Epilobietea angustifolii } \\
\hline Calamagrostis epigeios & + & $\mathrm{r}$ & . & . & . & . & + & . & 2.3 \\
\hline Rubus idaeus & + & + & 2 & . & + & . & + & . & 2.2 \\
\hline Bromus benekenii & . & . & . & . & + & + & . & . & . \\
\hline Sambucus nigra (c) & . & + & . & . & + & + & . & $\mathrm{r}$ & $\mathrm{r}$ \\
\hline Populus tremula & . & $\mathrm{r}$ & . & . & . & . & . & . & $\mathrm{r}$ \\
\hline \multicolumn{10}{|l|}{ VII. Others } \\
\hline Pinus sylvestris (a1) & . & . & . & . & 1.1 & 3.3 & . & 2.1 & . \\
\hline Quercus petraea (a1) & . & . & . & . & 1.1 & 1.1 & . & . & . \\
\hline Quercus robur (a1) & 2.1 & 1.1 & . & 3.3 & 4.3 & 2.2 & 2.2 & . & . \\
\hline Crataegus laevigata (b) & . & . & . & . & + & . & . & 1 & . \\
\hline Quercus petraea (c) & + & $\mathrm{r}$ & . & . & . & . & . & . & . \\
\hline Crataegus monogyna (c) & . & . & . & r & r & . & . & . & $\mathrm{r}$ \\
\hline Pinus sylvestris (c) & . & . & $\mathrm{r}$ & . & . & . & $\mathrm{r}$ & . & $\mathrm{r}$ \\
\hline Betula pendula (c) & . & $\mathrm{r}$ & . & . & . & . & . & . & $\mathrm{r}$ \\
\hline Larix cfr. kaempferi (c) & . & . & $\mathrm{r}$ & . & . & . & $\mathrm{r}$ & . & $\mathrm{r}$ \\
\hline Dryopteris carthusiana & . & $\mathrm{r}$ & + & . & + & 1.1 & . & $\mathrm{r}$ & $\mathrm{r}$ \\
\hline Luzula pilosa & + & . & $\mathrm{r}$ & . & . & . & . & . & . \\
\hline Maianthemum bifolium & $\mathrm{r}$ & + & $\mathrm{r}$ & . & + & + & . & 1.1 & + \\
\hline Moehringia trinervia & + & + & 1 & . & . & . & . & . & . \\
\hline Mycelis muralis & $\mathrm{r}$ & + & + & . & . & . & . & . & $\mathrm{r}$ \\
\hline Oxalis acetosella & + & + & + & . & . & + & . & $\mathrm{r}$ & + \\
\hline Athyrium filix-femina & . & . & . & $\mathrm{r}$ & . & . & + & . & . \\
\hline Chenopodium album & . & $\mathrm{r}$ & $\mathrm{r}$ & . & . & . & . & . & $\mathrm{r}$ \\
\hline Conyza canadensis & . & . & $\mathrm{r}$ & . & . & . & $\mathrm{r}$ & . & . \\
\hline Dryopteris dilatata & . & . & . & . & + & + & . & . & . \\
\hline Hieracium sabaudum & $\mathrm{r}$ & . & . & $\mathrm{r}$ & . & . & . & . & . \\
\hline Juncus effusus & . & . & $\mathrm{r}$ & . & . & . & + & . & $\mathrm{r}$ \\
\hline Plantago major & $\mathrm{r}$ & . & . & . & . & . & $\mathrm{r}$ & . & . \\
\hline Solidago virgaurea & $\mathrm{r}$ & + & . & . & . & . & . & . & . \\
\hline Brachythecium rutabulum & & $\mathrm{r}$ & $\mathrm{r}$ & . & . & . & . & . & . \\
\hline Dicranella heteromalla & $\mathrm{r}$ & . & $\mathrm{r}$ & . & . & . & . & $\mathrm{r}$ & . \\
\hline Hypnum cupressiforme & . & . & $\mathrm{r}$ & $\mathrm{r}$ & $\mathrm{r}$ & . & $\mathrm{r}$ & $\mathrm{r}$ & $\mathrm{r}$ \\
\hline
\end{tabular}

Sporadic species - II: Fagus sylvatica (b) 6(+); III: Carpinus betulus (b) 4(1.1); IV: Acer platanoides (a1) 4(1.1); Corylus avellana (b) 8(2.1); Fraxinus excelsior (c) 6(+); Paris quadrifolia 6(r); Sanicula europaea 4(+); Rumex sanguineus 7(r); Stellaria nemorum 1(r); Ribes spicatum $2(\mathrm{r})$; V: Arctium tomentosum 9(r); Arctium minus 7(r); Artemisia vulgaris 7(r); Cirsium vulgare 7(r); VI: Fragaria vesca 6(+); Gnaphalium sylvaticum $7(\mathrm{r})$; Sambucus nigra (b) 5(+); Centaurium erythraea 7(r); Salix caprea (b) 7(+); VII: Cornus sanquinea (b) 6 (+); Crataegus laevigata (c) 8(r); Quercus robur (a2) 8(2.2); Quercus rubra (b) 6(+); Quercus rubra (c) 6(r); Betula pendula (a2) 8(1.1); Betula pendula (b) 7(+); Achillea millefolium 9(r); Cerastium holosteoides 3(r); Ajuga reptans 3(+); Astragalus glycyphyllos 3(r); Holcus mollis 3(r); Epilobium adnatum 1(r); Equisetum arvense 3(r); Hypericum maculatum 7(r); Hypericum perforatum 9(r); Lathyrus niger 4(r); Lithospermum officinale 5(r); Prunus cerasifera (c) 8(r); Pyrus communis (c) 1(r); Pseudotsuga menziesii (a1) 7(2.2); Ranunculus repens 6(r); Ribes uva-crispa 8(r); Trifolium campestre 3(r); Trifolium medium $7(+)$; Veronica chamaedrys $7(\mathrm{r})$; Veronica officinalis $1(+)$; Vicia dumetorum 5(r); Viola mirabilis 9(r); Vicia sp. 3(r); Rubus sp. 3(+); Anemone sp. 3(r); Aulacomium androgynum 3(r); Polytrichastrum formosum 6(r); Sciuro-hypnum oedipodium 2(r); Pohlia nutans 1(r); Plagiothecium sp. 6(r).

Abbreviation: ex - exiguous.

$\left(17^{\circ} 00^{\prime} \mathrm{E} 52^{\circ} 38^{\prime} \mathrm{N}\right)$. The division is situated ca $2 \mathrm{~km}$ $\mathrm{N}$ of the Kąty village. Hordelymus europaeus grows in a deciduous forest stand under artificial regeneration, where many mature trees had been harvested and Fagus sylvatica seedling was planted. Hordelymus europaeus occupies an area of $5 \mathrm{~m}^{2}$, where ca 250 shoots were counted. Specimens are very robust, shoots with inflorescence range from $83 \mathrm{~cm}$ to $154 \mathrm{~cm}$, racemes range from $7.5 \mathrm{~cm}$ to $12 \mathrm{~cm}$ (Table 2).

3. Pniewy Forest District, Bytyń Forest Subdistrict

The oldest known locality in the Wielkopolska region. It was announced by RAFALSKI \& URBAŃSKI
(1932) on the basis of specimens collected in "a deciduous forest near Bytyń" by Kulesza (6.06.1931; POZ) and by Krawiec (26.06.1931; POZ). BIELAWSKA \& Bodniak $(1956,1959)$ specified, that species occurred quite often in four divisions (Fig. 1).

Current studies show, that $H$. europaeus occurs in extensive areas of oak-hornbeam forest (Galio sylvatici-Carpinetum). It was found in 23 divisions (Fig. 1), mostly in western part of the Bytyński Forest. Stands with wood barley represent diverse degrees of naturalness. Patches that are located in divisions 294 and 285 are protected (nature reserves "Brzęki przy starej 
Table 2. Morphological characteristics of Hordelymus europaeus in the Buczyna (Bu), Długa Goślina (DG) and Bytyń (By) Forest Subdistricts. To check up the position of particular locality (1A, 1B etc.) see Figure 1

\begin{tabular}{|c|c|c|c|c|c|}
\hline $\begin{array}{c}\text { Locality } \\
\text { Code (subdistrict) }\end{array}$ & $\begin{array}{l}\text { Approximate no } \\
\text { of shoots }\end{array}$ & $\begin{array}{l}\text { Shoot with inflores- } \\
\text { cence height }\end{array}$ & Raceme length & Middle leaf length & Middle leaf width \\
\hline $1 \mathrm{~A}(\mathrm{Bu})$ & 700 & $\begin{array}{c}37-83.5 \\
(65.4 \pm 0.31)\end{array}$ & $\begin{array}{c}4.5-10 \\
(7.35 \pm 1.3)\end{array}$ & $\begin{array}{c}10-28.5 \\
(22.32 \pm 3.77)\end{array}$ & $\begin{array}{c}0.4-0.9 \\
(0.69 \pm 0.11)\end{array}$ \\
\hline 1B (Bu) & 450 & $\begin{array}{c}43-128 \\
(81.28 \pm 23.19)\end{array}$ & $\begin{array}{c}5-11 \\
(7.47 \pm 1.37)\end{array}$ & $\begin{array}{c}11-32 \\
(22.27 \pm 5.08)\end{array}$ & $\begin{array}{c}0.5-1 \\
(0.74 \pm 1.15)\end{array}$ \\
\hline 2 (DG) & 250 & $\begin{array}{c}83-154 \\
(116.27 \pm 20.42)\end{array}$ & $\begin{array}{c}7.5-12 \\
(9.43 \pm 1.38)\end{array}$ & $\begin{array}{c}20-34 \\
(25.05 \pm 3.46)\end{array}$ & $\begin{array}{c}0.5-1.5 \\
(1.02 \pm 0.27)\end{array}$ \\
\hline 3C (By) & scattered & $\begin{array}{c}44.5-75 \\
(59.08 \pm 6.4)\end{array}$ & $\begin{array}{c}4.5-55 \\
(8.13 \pm 8.91)\end{array}$ & $\begin{array}{c}16-29 \\
(23.22 \pm 2.9)\end{array}$ & $\begin{array}{c}0.8-1.5 \\
(1.13 \pm 0.16)\end{array}$ \\
\hline 3E (By) & scaterred & $\begin{array}{c}40-100 \\
(72.2 \pm 14.84)\end{array}$ & $\begin{array}{c}5.5-10 \\
(7.47 \pm 1.24)\end{array}$ & $\begin{array}{c}12.5 \\
(21.77 \pm 4.74)\end{array}$ & $\begin{array}{c}0.5-1.4 \\
(0.99 \pm 0.22)\end{array}$ \\
\hline 3F (By) & 450 & $\begin{array}{c}74-131 \\
(107.78 \pm 16.35) \\
\end{array}$ & $\begin{array}{c}6-12 \\
(8.4 \pm 1.43) \\
\end{array}$ & $\begin{array}{c}16-35 \\
(24.65 \pm 4.84)\end{array}$ & $\begin{array}{c}0.7-1.5 \\
(1.1 \pm 0.23) \\
\end{array}$ \\
\hline ANOVA (F) & & $61,59 * * *$ & 1.31 & $3.13^{*}$ & $25.92 * * *$ \\
\hline
\end{tabular}

${ }^{*} \mathrm{p}<0.05 ;{ }^{* * *} \mathrm{p}<0.001$.

Gajówce" and "Bytyńskie Brzęki"). Under the dense canopy cover, the scarce clumps (ca 5-10 shoots) of $H$. europaeus grow scattered throughout the whole forest area (Table 1; 3A, 3B, 3C). Wood barley grows more abundant under a thinner canopy (Table 1; 3D, $3 \mathrm{E})$ whereas in a harvested stand with canopy gap we found rich agglomeration (ca 450 shoots) of this grass (Table $1 ; 3 \mathrm{~F}$ ). The lengths of shoots of Hordelymus vary considerably and range from $40 \mathrm{~cm}$ to $131 \mathrm{~cm}$ (Table 2). The highest shoots had specimens clustered in canopy gaps (3F), whereas the lowest were specimens growing in deep shadow (3C). The raceme was $4-12 \mathrm{~cm}$ long and did not differ among particular populations.

4. Kaczory Forest District, Zielonagóra Forest Subdistrict

Locality was reported by Kępczyński \& PeplińsKA (1993). Hordelymus europaeus was characterised as rare species in the Zielonagóra Forest Subdistrict and occurred in two divisions (Fig. 1).

In 2015 we searched the forest area between Osiek nad Notecią, Bąkowo and Dębowa Góra however no specimens of wood barley were found.

\section{CONCLUSION}

At present, the occurrence of Hordelymus europaeus has been confirmed in three forest districts of the Wielkopolska region: Buczyna, Długa Goślina and Bytyń. Hordelymus europaeus is widespread and numerous, only in the Bytyn Forest, where the population is stable and not endangered. In remaining localities populations are clearly smaller, composed of singular isolated aggregations, and as a consequence, its persistence in the future is much more uncertain.

Hordelymus europaeus is a shade tolerant species. Under the dense canopy of deciduous trees it forms small clumps (KęPCZYŃSKI \& PePLiŃSKA 1983, GŁOWACKI \& ZAŁUSKI 1997). Our observations showed, however, that lighting up a forest floor by selected harvest or even small clearcutting can stimulate the growth of wood barley and increase its abundance. Hence, it seems that sustainable silvicultural management does not negatively affect this grass.

\section{ACKNOWLEDGEMENTS}

We would like to thank mgr Paweł Urbański for mosses designation. The authors would like to thank dr Piotr Górski, as well as anonymous reviewers for their suggestions and comments made on the earlier version of the manuscript. The study was supported by the Department of Botany, the Poznań University of Life Sciences.

\section{REFERENCES}

Barć A., Brzustewicz M. (2006): Distribution of Hordelymus europaeus (L.) Jess. ex Harz in forest communities of the Beskid Mały Mountains. Biodiversity: Research and Conservation 3-4: 311-313.

BielawsKa A., BodNiak H. (1956): Rzadsze rośliny naczyniowe Lasu Bytyńskiego w pow. szamotulskim. Sprawozdania Polskiego Towarzystwa Przyjaciół Nauk 22(3): 1-44.

Bielawska A., Bodniak H. (1959): O utworzeniu rezerwatów w Lesie Bytyńskim. Przyroda Polski Zachodniej 3,1-2(7-8): 139-140.

GŁowaCKI Z., ZaŁUSKI T. (1997): Seminal and Cryptogamous plants. In: J.B. Faliński, W. Mułenko (eds). Seminal and Cryptogamous plants in the forest communities of Białowieża National Park. Ecological Atlas. Phytocoenosis 9 (N. S.) Supplementum Cartographiae Geobotanicae 7: 42.

Jackowiak B., Celka Z., Chmiel J., Latowski K., ŻUKOWSKI W. (2007): Red list of vascular flora of Wielkopolska (Polska). Biodiversity: Research and Conservation 5-8: 95-127. 
KęPCZyŃski K., Peplińska B. (1993): Szata roślinna leśnictwa Zielonagóra. Acta Universitatis Nicolai Copernici, Biologia 46(89): 29-109.

Klimko M., NowińsKa R., Czarna A. (2015): Epidermal micromorphology of Hordelymus europaeus (L.) Jess. ex Harz (Poaceae). Steciana 19(2): 89-96.

Matuszkiewicz W. (2001): Przewodnik do oznaczania zbiorowisk roślinnych Polski. Wydawnictwo Naukowe PWN, Warszawa.

Meusel H., Jäger E., Weinert E. (1965): Vergleichende Chorologie der zentraleuropäischen Flora. Bd. 1. Fischer Verlag, Jena.

Mirek Z., PięKoś-Mirkowa H., Zając A., ZająC M. (2002): Flowering plants and pteridophytes of Poland. A checklist. Vol. 1. Biodiversity of Poland. - Krytyczna lista roślin naczyniowych Polski. T. 1. Różnorodność biologiczna Polski. W. Szafer Institute of Botany, Polish Academy of Sciences, Kraków.

Mizianty M. (2001): The Agropyron-Elymus complex (Poaceae) in Poland: occurrence of Hordelymus europaeus. In: L. Frey (ed.). Studies on the grasses in Poland. W. Szafer Institute of Botany, Polish Academy of Sciences, Kraków: 161-176.

Mizianty M., Bieniek W., Czech A., StrząKa W., SzKLARCZYK M. (2006): Variability and structure of natural populations of Elymus caninus (L.) L. and their possible relationship with Hordelymus europaeus (L.) Jess. ex Harz as revealed by ALFP analysis. Plant Systematic and Evolution 256: 193-200.

Mizianty M., Szczepaniak M. (1997): Remarks on the Agropyron-Elymus complex (Poaceae) with special reference to its representatives in Poland Fragmenta Floristica et Geobotanica 42(2): 215-225 .

OBerdorfer E. (1990): Pflanzensoziologische Exkursionsflora. Ulmer Verlag, Stuttgart.

Ochyra R., Żarnowiec J., BeDnareK-Ochyra H. (2003): Census catalogue of polish mosses. Biodiversity of Poland. Vol. 3. W. Szafer Institute of Botany, Polish Academy of Sciences, Kraków.

RAFALSKI J., URBAŃSKI J. (1932): Rezultaty wycieczek florystycznych po Wielkopolsce. Wydawnictwo Okręgowego Komitetu Ochrony Przyrody na Wielkopolskę i Pomorze w Poznaniu 3: 46-49.

ZAJĄC A., ZAJĄC M. (eds) (2001): Distribution atlas of vascular plants in Poland. Laboratory of Computer Chorology, Institute of Botany, Jagiellonian University, Cracow.

For citation: Nowińska R., CZARnA A. (2015): Occurrence of Hordelymus europaeus (L.) Jess. ex Harz (Poaceae) in the Wielkopolska region. Steciana 19(4): 239-244. doi: 10.12657/steciana.019.024 